\title{
Environmental and Pathogenic Factors Inducing Brown Apical Necrosis on Fruit of English (Persian) Walnut
}

\author{
Michele Scotton, Enrico Bortolin, Antonio Fiorin, and Alessandra Belisario
}

First author: Department of Agronomy Foods Natural Resources Animals and Environment, University of Padova, Viale dell'Università 16, 35020 Legnaro, Padova; second author: Nogalba, Consorzio Produttori di Noce, 45010 Pettorazza, Rovigo, Italy; third author: Il Noceto, Società Consortile s.r.l., 31040 Chiarano, Treviso, Italy; and fourth author: Consiglio per la Ricerca in Agricoltura e l'analisi dell'economia agraria-Centro di Ricerca per la Patologia Vegetale (CRA-PAV), Via C. G. Bertero 22, 00156 Roma, Italy.

Accepted for publication 17 July 2015.

\begin{abstract}
Scotton, M., Bortolin, E., Fiorin, A., and Belisario, A. 2015. Environmental and pathogenic factors inducing brown apical necrosis on fruit of English (Persian) walnut. Phytopathology 105:1427-1436.

Brown apical necrosis (BAN) is a most recently described disease affecting English (Persian) walnut fruit. BAN was only recorded in intensively managed walnut orchards and was found to be a disease complex mainly caused by Fusarium species. All fungi associated with this disease are polyphagous and ubiquitous, not specific to walnut. Consequently, BAN occurrence is more strictly dependent, than generally, on the interaction between pathological features and environmental conditions. Environmental variables identified with regression analysis showed that maximum temperature, angle of main wind direction versus

tree row orientation, and orchard distance to the closest river/canal, all representative of climatic conditions occurring in the orchard, were related to fruit drop. The factor displaying the highest influence on severity of BAN fruit drop was maximum temperature and only subordinately factors are associated with relative humidity. BAN symptoms were reproduced with in planta artificial inoculation, and fruit drop of symptomatic fruit was significantly higher than that of the noninoculated trees for each type of inoculum (Fusarium semitectum, F. graminearum, and Alternaria spp.). F. semitectum and $F$. graminearum were more aggressive than Alternaria species, and the earliest artificial inoculations in mid-May resulted in the highest fruit drop. The extension of walnut fruit susceptibility and the conducive environmental factors to BAN are discussed.
\end{abstract}

"English" or "Persian" walnut (Juglans regia L.) is the most commercially cultivated walnut species worldwide, being used for fruit (edible nuts) or timber production. In the last 20 years, increasing cultivation in Italy and southern and eastern Europe has been accompanied by changes in cultural management, varieties, and extension of land-use. In order to increase fruit quality and yield and to reduce labor requirements, plantings have changed to highly specialized commercial cultivation with intensive pruning, fertilization, irrigation, and chemical treatments. Hedgerow-trained orchards represent the most intensive management of walnut fruit production in northern Italy and southern France since 1991. For these reasons, maintaining insect and especially disease free conditions is an important aspect of quality and yield. Among diseases that affect walnut fruit, brown apical necrosis (BAN) is the most recently described (Belisario et al. 2002). BAN was first observed in several Po Valley Italian orchards in 1998. Its name is typical of its symptoms, originating exclusively at the fruit stigmatic or blossom end (Fig. 1), consisting of a brown to dark-brown spot enlarging to a patch, then enlarging up to two-thirds of the fruit surface. The style is the organ through which disease expands to the fruit inner tissues (Belisario et al. 2002). Diseased fruit show a brown to blackish rot of inner tissues (Fig. 1). BAN results in premature fruit drop, with losses up to $40 \%$ in severe years. From 1999 onwards, BAN has been observed both in Italy and in southern France, and its severity was notable for the following 2 years (1999 to 2000) (Belisario et al. 2001, 2002). The disease has been recorded exclusively in commercial, intensively managed walnut orchards 5 years old and older (Belisario et al. 2010). BAN has been observed

Corresponding author: A. Belisario; E-mail address: alessandra.belisario@entecra.it

http://dx.doi.org/10.1094/PHYTO-01-15-0029-R

(C) 2015 The American Phytopathological Society infecting cultivars Lara, Pedro, Chandler, and Sunland (Belisario et al. 2001, 2002).

Of the over 10 species of Fusarium isolated from BAN-affected fruit, $F$. graminearum and $F$. semitectum were the most frequently isolated. Artificial inoculations performed on detached walnut host materials confirmed the association of Fusarium spp. with BAN (Belisario et al. 2002). Within the genus Alternaria, three species belonging to the small-spored catenulate taxa related to A. alternata (A. alternata, A. arborescens, and A. tenuissima) were found pathogenic on walnut and involved in BAN (Belisario et al. 2004; Hong et al. 2006). Thus, BAN is considered a disease complex in which several fungi are involved, but mainly Fusarium species. Since previous artificial inoculations were made on detached host materials, it was not possible to observe symptom progression to outer and inner walnut fruit tissue as the host material deteriorated in 2 weeks after inoculation (Belisario et al. 2002).

BAN is a disease complex similar to other disease complexes caused by Fusarium spp., e.g., pumpkin fruit rot (Elmer 1996), asparagus decline (Borrego-Benjumea et al. 2014), and wheat foot rot, crown rot, or head blight (Klix et al. 2008; Parry et al. 1995), in which Fusarium species composition differ with geographical area and over time. Changes in fungal frequency and dominance may indicate the importance of environmental and biological factors in disease development. Moreover, all fungi associated with BAN are polyphagous and ubiquitous, i.e., not specific to walnut. Consequently, BAN occurrence is likely more dependent on interaction between pathological features and environmental conditions. As a general consideration, to point out the cause(s) of a disease, the pathogen's lifecycle should be linked to (i) several environmental variables (i.e., temperature, rain, humidity, and frost) that may trigger multiplication and spreading of the pathogen; (ii) agronomic features (i.e., soil characteristics and irrigation); and (iii) host plant. 
Commonly, the ecological influences on the occurrence and severity of the disease can be numerous.

The aims of the present work were to (i) identify the environmental factors (geographical, site, management, and weather) conducive to the disease; (ii) reproduce the whole symptomatology and monitor the development of BAN on inoculated fruit in the field; and (iii) understand the period of high susceptibility of walnut fruit to the disease.

\section{MATERIALS AND METHODS}

Environmental factors associated with BAN natural infection. Research was conducted in eastern Po Valley commercial walnut orchards located in the Veneto Region belonging to Nogalba
(Rovigo area) and Il Noceto (Treviso-Venice area), Walnut Producers Cooperatives, where natural BAN infection was initially detected, and in the Tor Mancina experimental farm close to Monterotondo (Roma Province, Latium Region) belonging to the CRA-Plant Pathology Research Center, where BAN was never detected, but reproduced by artificial infections (Fig. 2). From 1998 to 2001, damage due to natural BAN infection was examined in 17 commercial, hedgerow-trained English walnut ('Lara') orchards located in Rovigo (nine orchards), and Treviso-Venice (eight orchards). These orchards (trees spaced $7 \times 3.5 \mathrm{~m}$ ) were between 7 and 12 years old growing in fertile, plain soils. The tree rows were herbicide-sprayed (glyphosate) and volunteer resident grass vegetation mowed three to four times per year in row middles. Irrigation utilized an under tree sprinkler system.

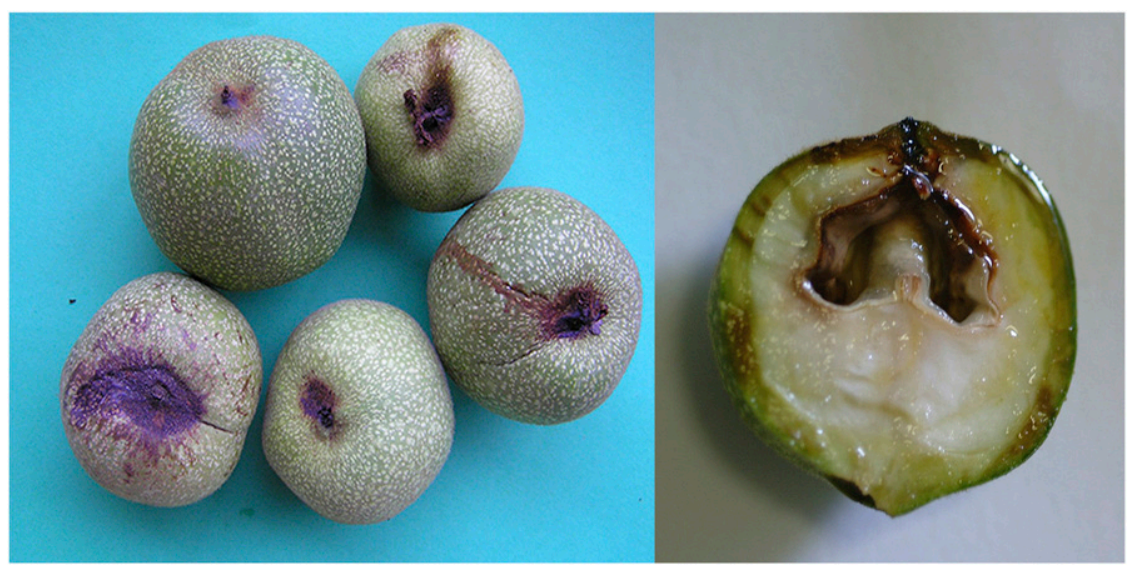

Fig. 1. Typical external symptoms of brown apical necrosis (BAN) at the stigmatic end of walnut fruit due to natural infection (left). Blackish degraded inner walnut fruit tissues caused by BAN from natural infection. The passage of the infection through the style is evident (right).

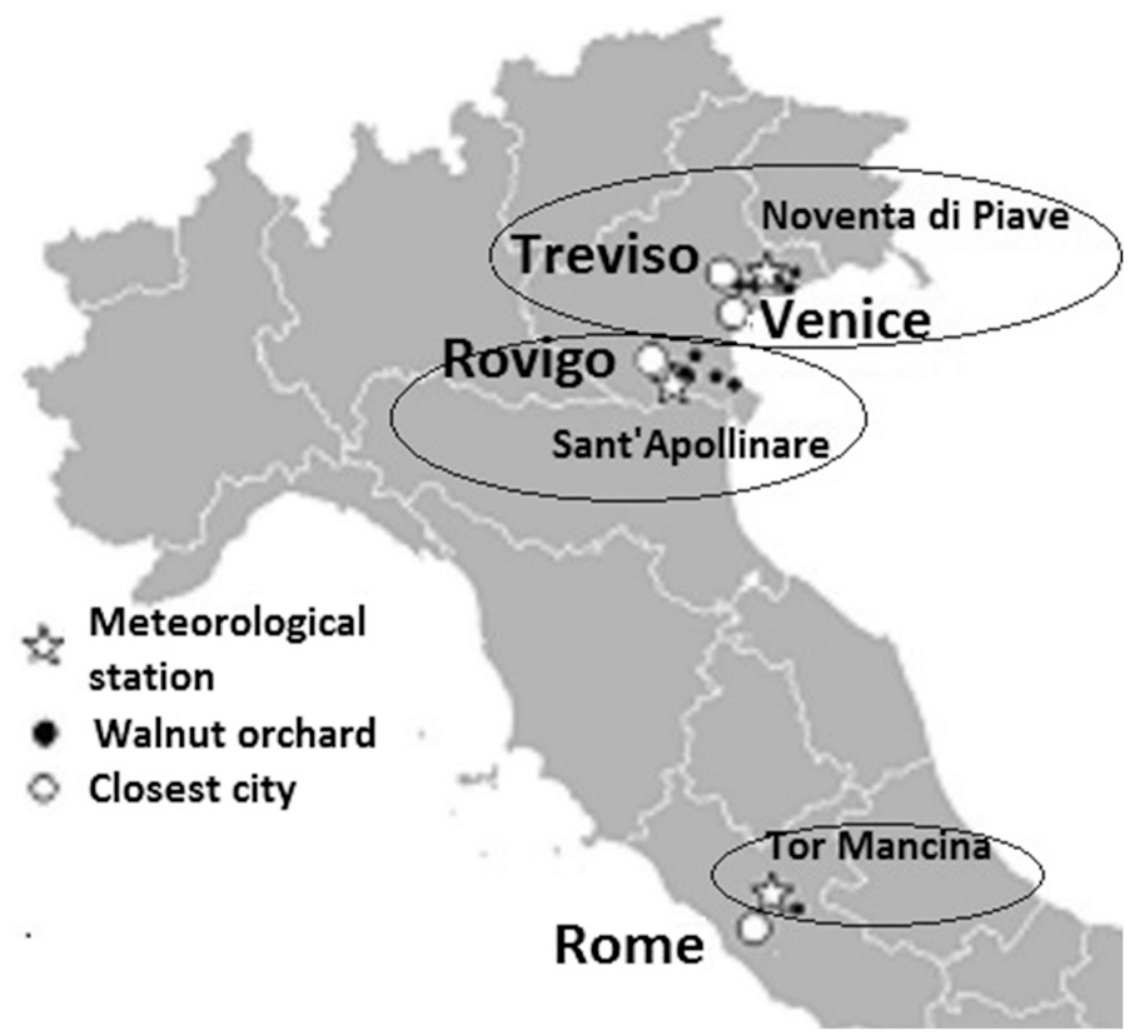

Fig. 2. Location of walnut orchards with brown apical necrosis (BAN) resulting from natural (years 1998 to 2001) and artificial infections (years 2002 to 2003 and 2007 to 2008) and reference meteorological stations (Sant'Apollinare, Noventa di Piave, and Tor Mancina). Ellipses group the orchards of the same geographical area and the corresponding reference meteorological station. 
In all 17 orchards located in Veneto Region, natural fruit drop was assessed at 14 sites in 1999 and 2000, at one site in 1998 and 1999, and at two sites in 2000 and 2001. The amount of fruit drop was estimated by counting the number of fruit harvested in relation to the total number of flowers/young fruit present at the beginning of the season. This practice is currently done each year in most of the commercial English walnut orchards and consists of a weekly counting the number of fruit from May to August on two branches of four trees randomly chosen in a given orchard.

Environmental factors were studied concerning their effect on occurrence and severity of BAN. Several orchard characteristics were considered: distance from the closest river or canal (DCR, only water courses wider than $5 \mathrm{~m}$ were considered), age of trees in the orchard, tree canopy cover on soil (\%, percentage of soil covered by the tree projection, obtained by multiplying walnut tree hedge width $\times$ total hedge length), total canopy volume $\left(\mathrm{m}^{3}\right.$, obtained by multiplying canopy height $\times$ canopy width $\times$ total hedge length), vigor (visually estimated in September within a scale from 1 to 5 on the basis of the length of 1-year shoots), and amount of irrigation water given to the trees till the date of survey (calculated by multiplying the total number of irrigation hours $\times$ hourly flow rate of the irrigation system). Data were provided for the studied orchards by technical advisers of the two walnut cooperatives. In addition, the following meteorological factors were considered: dominant wind direction; leaf wetness; solar irradiation; rainfall; minimum, maximum, and mean air temperature $\left({ }^{\circ} \mathrm{C}\right)$ (MinT, MaxT, and MeanT); and relative humidity. Daily values of meteorological variables, measured at the height of $2 \mathrm{~m}$ above the soil, were provided by ARPAV (Agenzia Regionale per la Prevenzione e Protezione Ambientale del Veneto) at the stations of Sant'Apollinare and Noventa di Piave (Fig. 2). Since wind effect on orchard microclimate strongly depends on its angle with the orientation of tree rows, a parameter named angle of main wind direction versus tree row orientation (AWR) was considered. This parameter was calculated each day on the basis of the orchard tree row direction and the daily value of the dominant wind direction measured in the meteorological station used as reference. It varied between 0 and $90^{\circ}$, where $0^{\circ}$ is when the dominant wind direction was parallel to the tree row and $90^{\circ}$ when the dominant wind direction was perpendicular to the tree row.

Etiology of BAN: Inoculations of walnut fruit in the field. To determine the role of fungal agents isolated from BAN- affected fruit and to reproduce the entire symptomatology, artificial inoculations were carried out in planta using proper inoculation methods described below.

Inoculum preparation and inoculation procedure. Fungal isolates used for artificial inoculations were obtained from BAN infected fruit collected in the area identified in previous work (Belisario et al. 2002). Consequently, inoculations were performed using the following fungal strains: F. semitectum ISPaVe1946, F. graminearum ISPaVe1945, and Alternaria small-spored catenulate species belonging to the Alternaria alternata complex. In this latter case, the strains A. alternata ISPaVe 1779, A. arborescens ISPaVe 1780, and A. tenuissima ISPaVe1770 were mixed and used as "Alternaria spp.". Those strains were determined to be pathogenic to walnut fruit in previous investigations (Belisario et al. 2001, 2002, 2004; Hong et al. 2006).

F. graminearum, F. semitectum, A. alternata, A. arborescens, and A. tenuissima single-spored isolates were subcultured on potato dextrose agar (PDA, Oxoid, Basingstoke, UK) and grown at $25^{\circ} \mathrm{C}$ in the dark for 7 to 10 days. Cultures were then flooded with agarized $(0.22 \%$ of technical agar, Oxoid) sterile distilled water (SDW), scraped and filtered through two layers of sterile cheesecloth. The concentration of conidial suspension was determined using a hemocytometer and adjusted with agarized SDW to $1 \times 10^{6}$ conidia/ml. For inoculum preparation of Alternaria spp., an equal volume of an agarized suspension of $1 \times 10^{6}$ conidia $/ \mathrm{ml}$ of each species was mixed to obtain a three species mixed conidial suspension (Alternaria bulk mix). Previous experiments carried out on paired cultures of the three Alternaria strains grown on PDA and on Czapek-Dox agar (Oxoid) confirmed absence of incompatibility or inhibition effects among them (data not shown).

For inoculations, a $10-\mu l$ droplet of conidial suspension was released on stigmas or on the stigmatic end of each walnut flower/fruit using an Eppendorf multipette plus (Eppendorf, Hamburg, Germany) (Fig. 3). When all flowers/fruit of one tree were inoculated, the entire tree was wrapped in a net to exclude fruit dropped contamination from bordering trees and prevent contamination by soilborne agents, such as Fusarium spp. Trees used as controls were treated following the same procedure described above except only agarized SDW was used.

Artificial inoculations and reisolations. In years 2002 and 2003, artificial inoculations were carried out in a commercial walnut orchard of 'Lara' located in Costa (Rovigo Province, Veneto

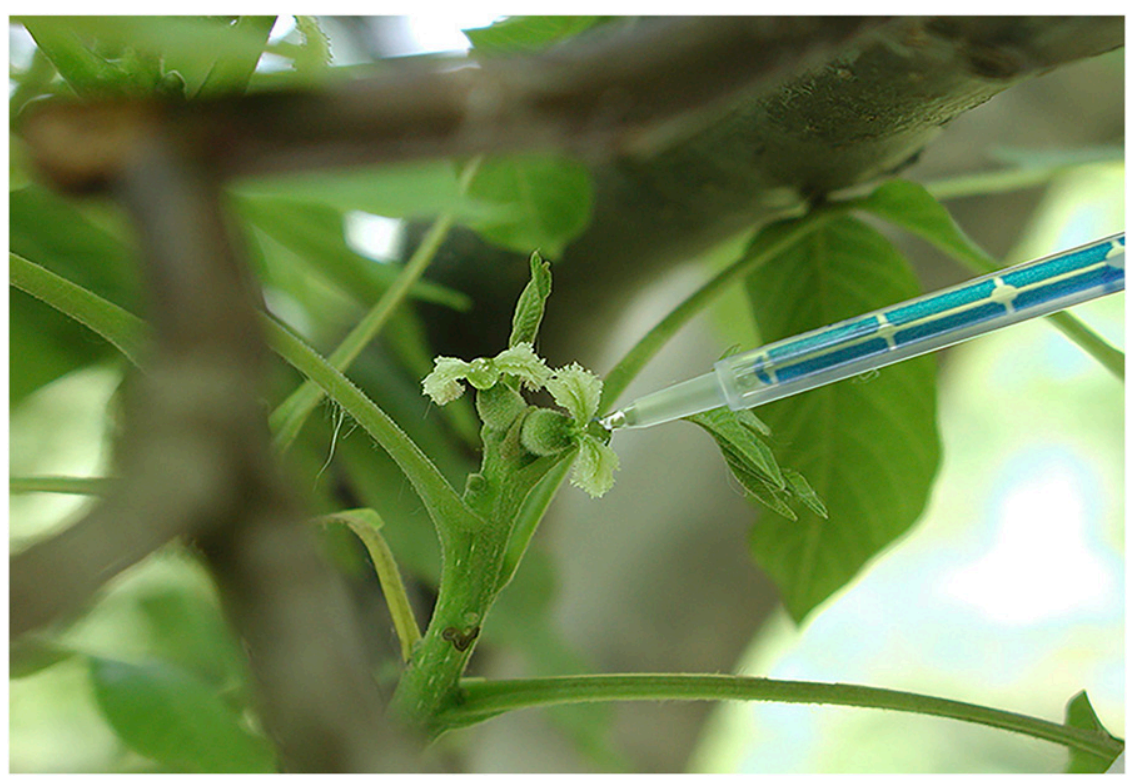

Fig. 3. Early inoculation (mid-May) performed by a multipette at a fixed volume of 10- $\mu 1$ droplet of conidial suspension released on stigmas of walnut flowers/fruit. 
Region), which was tested positive for BAN in previous years. Alternaria spp., F. graminearum, and F. semitectum inocula were used to fulfill Koch's postulates. A fourth noninoculated treatment was used as a control. For each treatment all flowers/fruit of a tree were inoculated. The flowers/fruit of each tree in the study were inoculated with a droplet of agarized conidial suspension (Fig. 3), as described above, and recorded per tree. Inoculations were performed at three different stages of the fruit development starting at flowerings on 14 May, 28 May, and 12 June 2002; and on 14 May, 28 May, and 11 June 2003. These phenological stages were chosen based on the information retrieved from previous experiments (Belisario et al. 2002) that showed the stigmas as the most receptive structure since they hold the conidia and favor their germination by keeping droplets of water. In general, walnut blooming is around mid-May, shell hardening and sealing is by the end of June, while harvesting generally start by the second half of September, though differences are present among varieties.

In 2007 and 2008, inoculations were carried out in an orchard of Tor Mancina farm on 'Lara' walnut trees on which BAN was never observed. At this site, the annual mean temperature is generally $1.5^{\circ} \mathrm{C}$ higher than that in Costa, mainly due to the higher temperatures between June and January, and rainfall ( $790 \mathrm{~mm}$ per year) have a typical Mediterranean distribution with a maximum during winter and a minimum rainfall in summer. The fungal strains and the inoculation procedure, used in this study, were similar to those applied in Costa. F. semitectum was tested in both years while Alternaria spp. only in 2008, and F. graminearum only in 2007 due to a shortage of walnut trees available. Inoculations were performed on 9 May, 23 May, and 6 June 2007; and on 16 May, 30 May, and 4 June 2008. Artificial inoculation trials were designed as a randomized complete block with four replicates. Each block consisted of plots having trees equal to the number of treatments. Each tree was inoculated just once and wrapped with a net as previously described.

Dropped fruit collected in the net fixed under each tree, and suspended above the soil, were analyzed in the laboratory. They were collected five times for the experiments conducted in Costa on 6 and 24 June, and on 1, 8, and 15 July in both years. For the third inoculation, the first date of sampling was not considered. In turn, for trials carried out in Tor Mancina two dates of fruit collection were considered, on 2 or 3 and 16 or 17 July for 2007 and 2008, respectively.

Fruit dropped from inoculated plants were visually inspected for BAN symptoms and a minimum of $20 \%$ of symptomatic fruit was subjected to isolation. Fruit dropped from trees used as controls in Costa were also assessed for the presence of BAN and, if symptomatic, they were subjected to fungal isolations as described below. Fruit dropped from trees used as controls in Tor Mancina did not show any symptoms of BAN. In this case, $10 \%$ of dropped fruit were randomly chosen for isolation. Fruit were thoroughly washed in tap water, surface disinfested in $1 \% \mathrm{NaOCl}$ for $30 \mathrm{~s}$ followed by $2 \times 30 \mathrm{~s}$ in sterile deionized water washes, and then dried on sterile filter paper in a laminar flow hood. From each nut, small pieces at the edge of discolored tissues were excised and plated on PDA or acidified PDA (Corpas-Hervias et al. 2006). Isolations were performed on whole or halved nuts mainly in correspondence to the style and pericarp, but also the ovary, and eventually the kernel were analyzed. Plates were incubated at room temperature $\left(22\right.$ to $24^{\circ} \mathrm{C}$ ) and examined daily for fungal growth. Fungal mats were subcultured on PDA or acidified PDA (APDA) and identified based on morphological characteristics. For the inoculations performed in Costa in 2002 and 2003, analyses were carried out also on fruit randomly collected from the tree to better understand initial symptoms of BAN and progression of the disease inside fruit tissue before eventual fruit drop. For each type of inoculum, 15 fruit were picked up randomly from the walnut tree for each treatment on 6 and 24 June, and on 1 and 15 July. Fruit were visually inspected for BAN symptoms and all symptomatic fruit were subjected to isolation following the same procedure described above.

Statistical analysis. To analyze the effect of geographical area, the 2-year means of fruit drop calculated for the 17 orchards were subdivided into the Rovigo and Treviso-Venice groups and subjected to parametric analysis of variance (ANOVA) and the same was done for all environmental variables. When necessary, values were previously transformed to meet parametric ANOVA assumptions. Pearson correlation coefficients were calculated between each environmental variable and fruit drop.

To identify the relationship between environmental factors and fruit drop severity in years 1998 to 2002, data were analyzed through multiple linear regression, model stepwise using PROC REG in SAS (Version 9.3, SAS Institute, Cary, NC). Possible error dependence, heteroscedasticity and collinearity problems were checked according to Durbin and Watson (1951), White (1980), and Belsley et al. (1980), with the DW, SPEC, and COLLIN options in the MODEL statement.

Meteorological variables used in the regression analysis were identified using a procedure aimed at finding the period during which weather conditions were more conducive to fruit drop. For each orchard, differences between years for eight weather variables were calculated. Values were then related (Pearson correlation) to differences between years of estimated fruit drop. Weather variables were calculated for each possible interval between 1 April and 30 September. Successively, in the regression analysis, only values of the eight weather variables calculated for the interval with the highest mean of the correlation coefficients were used.

To demonstrate relative importance of environmental factors, regression analysis was performed in two ways. First, the original yearly values of the percentage of fruit drop (dependent variable, Y) and all available environmental variables (independent variables, $\mathrm{X})$ were taken into account. In this way, assessment of the relative importance of all available variables was possible. The second approach analyzed effects of weather variability between years: differences between the first and second year, in terms of percentage fruit drop $(\mathrm{Y})$ and of the meteorological variables $(\mathrm{X})$, were taken into account. In both types of analyses, first order (original independent variables) and second order (interactions) terms were used.

For the artificial inoculation experiments, percentage of BAN symptomatic dropped fruit related to inoculated flowers/fruit was calculated for each date of collection. As stated above, in experiments performed in Tor Mancina, artificial inoculation with Alternaria spp. was not performed in 2007, and with F. graminearum in 2008, due to shortage of plant material. In a preliminary ANOVA of Tor Mancina data, it was found that the effect of the factor year did not significantly affect fruit drop in the two inoculation treatments available for both years (F. semitectum and noninoculated control). Therefore, those values were averaged and the factor experiment was considered for only three levels (Costa 2002, Costa 2003, and Tor Mancina 2007 to 2008). To highlight the effect of the type of inoculum in each experiment, the total percentages of all or symptomatic fruit dropped within the last date of collection (15th of July), averaged by inoculation time, were subjected to one-way ANOVA. As none of the possible transformations produced values matching the parametric ANOVA requirements for treatment variance homogeneity, the nonparametric approach of the Monte Carlo permutation test was chosen. According to Osborne (2010), the values were square-root transformed to eliminate the strong mean-variance relationship. Where ANOVA results were significant, the percentage of dropped fruit recorded for the four types of inoculum was compared with three orthogonal contrasts (fungi versus control, Fusarium spp. versus Alternaria spp., F. graminearum versus $F$. semitectum).

To determine whether the drop of symptomatic fruit decreased in relation to inoculation time, inoculation dates were converted to 
number of days from 1/5 and linear or power regression analysis were performed using SAS procedures REG and NLIN.

Data obtained from reisolations performed on artificially inoculated, symptomatic fruit either dropped or randomly picked up from the tree were also subjected to nonparametric ANOVA according to a three-factor model (type of inoculum, inoculation time, and experiment). Probabilities for main effects were generated by restricting permutations within levels of the other factors (Edgington and Onghena 2007). Interactions were tested through not-restricted permutations after residuals calculation (Anderson and Ter Braak 2003). According to Pillar De Patta (2006), nonparametric ANOVA was followed by post-hoc pairwise group ANOVAs to compare group means, when significant. The test criterion for the main effects and two-group contrasts was among groups sum of squares $(\mathrm{Qb})$ : for the interactions, the pseudo F-ratio ( $F=Q b / Q w$, where $Q w$ is the within groups sum of squares) was used. Tests were carried out with software Multiv (Pillar De Patta 2006).

\section{RESULTS}

Environmental factors associated with BAN natural infection. Climate of Rovigo and Treviso-Venice areas showed similar thermic conditions (mean annual temperature $13.3^{\circ} \mathrm{C}$ and yearly temperature range $20.3^{\circ} \mathrm{C}$ ). Rainfall had equinoctial distribution, but was significantly lower in the Rovigo area $(714 \mathrm{~mm}$ versus $956 \mathrm{~mm})$.

During 1998 to 2001, the average fruit drop was 23\%, and mean fruit drop was significantly higher in the Rovigo area $(33 \%)$ compared with Treviso-Venice (12\%) (Table 1). In the orchards where fruit drop was measured both in 1999 and 2000 (14 sites out of 17), fruit drop was considerably higher in 2000 (25.7\%) than in $1999(19.3 \%)$. The seasonal interval with highest correlation among weather variables and between years damage differences was 24 May to 21 June (mean of the coefficients 0.481). In that period, correlation was highest for MaxT (0.80), followed by relative humidity (0.58) and solar irradiation (0.56). Coefficient means were mostly above 0.35 for interval before 24 May, while they dropped below this value for intervals after 24 May.

Meteorological characteristics more strictly correlated with fruit drop were angle of main wind direction versus tree row orientation (AWR), mean temperature (MeanT), and maximum temperature
(MaxT). Among site/orchard/management characteristics, DCR, and total canopy volume showed the highest values of the correlation coefficients (Table 1).

Analyses of multiple linear regression on the yearly fruit drop values collected from 1998 to 2001 performed against all variables considered, identified the interaction AWR $\times$ relative humidity, followed by MaxT, and the interaction DCR $\times$ vigor, as the most significant variables (Table 2 ). Total variability explained by this model was $60.9 \%$. MaxT explained $18.1 \%$ of the variability; the positive relationship with fruit drop was mainly related to higher temperatures in 2000 (MaxT $28.2^{\circ} \mathrm{C}$ compared with $27.5^{\circ} \mathrm{C}$ in 1999) and this may also give an explanation to the higher fruit drop recorded in Rovigo area where temperatures were on average higher than in Treviso-Venice area $\left(27.85\right.$ versus $\left.27.72^{\circ} \mathrm{C}\right)$.

In the regression analysis, performed by taking into account only differences between first and second year fruit drop in the same orchard, the total data variability decreased by $85 \%$ ( 862 versus 5636; Table 2). This analysis identified MaxT as the most important explanatory parameter followed by rainfall (Table 2), with a total amount of explained variability of $72 \%$. For both variables, the relationship with fruit drop was positive.

BAN etiology: Fruit drop, progression of symptoms, and recovery of fungi. Generally, BAN symptoms were successfully reproduced on inoculated fruit. Fruit drop of inoculated, symptomatic fruit was significantly higher compared with those from noninoculated trees for each type of inoculum used and in both locations, and both Fusarium species were more aggressive than Alternaria spp. (Table 3). The early artificial inoculations induced highest fruit drop (Fig. 4). Fruit drop gradually decreased either for later inoculations or for later collection dates. For inoculations performed in May, fruit drop was particularly high immediately after inoculation. This trend was particularly evident in Costa 2003 and in Tor Mancina experiments, but less evident in Costa 2002. Total drop of BAN symptomatic fruit decreased from the first to the last inoculation date following linear or power trends (Fig. 5). Differences in fruit drop between inoculation treatments and control were particularly high for the first inoculation date and for Fusarium inocula.

For experiments conducted in Costa, F. graminearum and $F$. semitectum resulted in the highest reproducibility of disease with fruit drop of 6.7 to $11.8 \%$ and 10.5 to $15 \%$, respectively (Table 3). The fungi were reisolated from 60 to $67.5 \%$ (2-year

TABLE 1. Fruit drop observed during 1998 to 2001 and site, type of management, and weather variables of 17 walnut orchards in the Po-Venetian plain ${ }^{z}$

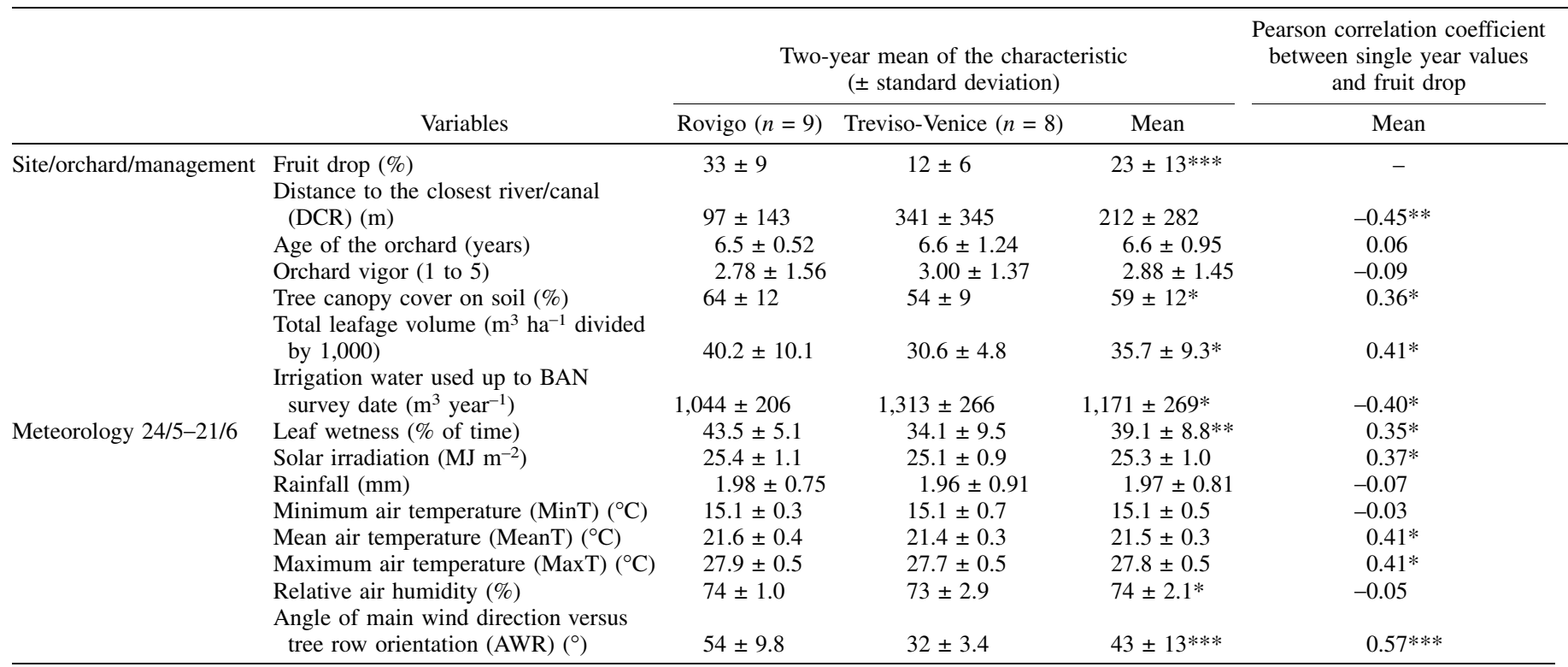

z Analysis of variance significance level: *, $0.01<P<0.05$; **, $0.001<P<0.01$; ***, $P<0.001$. 
average) of the symptomatic fruit (Table 4). A similar trend in recovery of $F$. graminearum and $F$. semitectum from symptomatic fruit was observed for all three inoculation times (Fig. 6). Percentage of reisolation did not vary significantly between years (Table 4). For Fusarium species, the lowest percentage of reisolation of dropped fruit was observed in the second half of June (24th of June) (Fig. 6). Alternaria spp. inoculations induced the drop of a small amount of symptomatic fruit ranging from 1.9 to $2.3 \%$ (Table 3 ), with peaks of reisolations up to $100 \%$ and an average value of $67.3 \%$ (Table 4). The curve obtained from reisolation data of Alternaria spp. artificial inoculations did not show a clear trend for both Fusarium species. This was probably due to a low number of diseased fruit available for reisolation (data not shown). In Costa, BAN was observed also on control trees but at a significant lower percentage of $\leq 1.0$ (Table 3 ) compared with the inoculated treatments, and the fungal pathogenic species of BAN were recovered from inoculated, diseased fruit (data not shown).

In Tor Mancina, results obtained with the different types of inocula by artificial infections were almost the same as those obtained in Costa, while symptomatic fruit drop was much higher than that in Costa (Table 3). Percentage of reisolation was similar to that obtained in Costa (Table 4). The first inoculation resulted in the highest level of disease. No diseased fruit were observed in the noninoculated control (Fig. 4). Recovery of the three fungi from diseased fruit showed a similar pattern over sampling dates (Fig. 6).

Results obtained from fruit randomly sampled from inoculated trees in Costa experiments showed that the highest percentages of symptomatic fruit developed in the first inoculation were 33,28 , and 25 for $F$. semitectum, F. graminearum, and Alternaria spp., respectively; while on control plants it was $8 \%$ (data not shown). For the second and third inoculation, percentages of diseased fruit ranged from 12 to 16 and 3 to 8 , respectively. From those symptomatic fruit, recovery of the fungi ranged from 20 to $100 \%$ (Table 4). The lowest recovery corresponded to the second half of June (24 June) (Fig. 6). On average, the recovery of the two Fusarium spp. was not significantly different, with $77 \%$ for $F$. semitectum and $63 \%$ for $F$. graminearum, but that of F. semitectum was significantly different from that of Alternaria spp. (55\%) (Table 4).

Visual inspection, after sectioning, of randomly sampled fruit showed the symptomatic phases of colonization and lesions of inner tissues never exceeded half the fruit length with apparent browning of the tissue, but absence of rot. Infection started from the style and extended inwards to the ovary tissues, particularly evident on the fruit inoculated with $F$. semitectum. In general, disease progression on the fruit in the third inoculation was slower than that on the fruit inoculated earlier, and about $50 \%$ of the inoculated fruit showed only style necrosis without further progression of infection. Alternaria spp. tended to progress straight into the ovary when inoculated in June. Browning of host tissues caused by F. semitectum and less by $F$. graminearum progressed faster in early inoculations than in later inoculations.

$F$. semitectum showed a necrotizing effect on the flowers/fruit, particularly in the first inoculation conducted in mid-May, inducing fruit drop within 20 days. Young flower/fruit tissues rapidly became brown and necrotic with a deformation and shriveling of the husk. F. graminearum and Alternaria spp. were less aggressive than F. semitectum.

TABLE 2. Parameters and intercept estimation, partial (in brackets) and model $r^{2}$, and total sum of squares of the multiple linear regressions between fruit drop percentage and environmental variables in 17 walnut orchards in the Po-Venetian area for the years 1999 to $2000^{2}$

\begin{tabular}{|c|c|c|c|}
\hline \multirow[b]{2}{*}{ Statistical parameter } & \multirow[b]{2}{*}{ Variable } & \multicolumn{2}{|c|}{ Type of values used in the regression analysis } \\
\hline & & Single year values & Differences between years \\
\hline \multirow[t]{6}{*}{ Parameter estimation (partial $r^{2}$ ) } & Maximum air temperature $(\operatorname{MaxT})\left({ }^{\circ} \mathrm{C}\right)$ & $11.9(0.181)$ & $13.4(0.636)$ \\
\hline & Rainfall (mm) & - & $2.53(0.083)$ \\
\hline & Interaction distance from the closest & & \\
\hline & river/canal $(\mathrm{DCR})(\mathrm{m}) \times$ vigor & $-0.00439(0.112)$ & $\mathrm{Nu}$ \\
\hline & $\begin{array}{l}\text { Interaction angle of main wind direction } \\
\text { versus tree row orientation }(\mathrm{AWR})\left(^{\circ}\right) \times\end{array}$ & & \\
\hline & relative humidity $(\mathrm{RH})(\%)$ & $0.00679(0.3166)$ & - \\
\hline Intercept estimation & & -328 & 0 \\
\hline Model $R^{2}$ & & 0.609 & 0.720 \\
\hline Total sum of squares & & 5636 & 862 \\
\hline
\end{tabular}

${ }_{\mathrm{z}}$ All parameters are significant at least at $P<0.02 . \mathrm{Nu}=$ not used in this regression analysis.

TABLE 3. Nonparametric analysis of variance (ANOVA) and orthogonal contrasts of symptomatic fruit drop (\%) of artificial inoculation experiments performed in two sites $^{\mathrm{x}}$

\begin{tabular}{|c|c|c|c|c|c|c|c|c|}
\hline \multirow[b]{3}{*}{ Experiment } & \multirow{2}{*}{\multicolumn{4}{|c|}{ Fungi used as inoculum }} & \multicolumn{4}{|c|}{ ANOVA (significance level) } \\
\hline & & & & & \multirow[b]{2}{*}{$\begin{array}{l}\text { All } \\
\text { groups }\end{array}$} & \multirow{2}{*}{$\begin{array}{l}\text { Contrast } \\
\text { fungi versus } \\
\text { control }\end{array}$} & \multirow{2}{*}{$\begin{array}{c}\text { Contrast } \\
\text { Fusarium spp. } \\
\text { vs. Alternaria }\end{array}$} & \multirow{2}{*}{\begin{tabular}{l}
\multicolumn{1}{c}{ Contrast } \\
F. graminearum vs. \\
F. semitectum
\end{tabular}} \\
\hline & $\begin{array}{c}\text { Fusarium } \\
\text { graminearum }\end{array}$ & F. semitectum & $\begin{array}{l}\text { Alternaria } \\
\text { bulk }\end{array}$ & Control & & & & \\
\hline Costa 2002 & 11.8 & 15 & 2.3 & 1 & 0.001 & 0.009 & 0.014 & 0.133 \\
\hline Costa 2003 & 6.7 & 10.5 & 1.9 & 0.9 & 0.001 & 0.026 & 0.015 & 0.107 \\
\hline Tor Mancina 2007-2008 & 28.7 & 32.3 & 15 & 0 & 0.002 & 0.005 & 0.136 & 0.109 \\
\hline Tor Mancina 2007-2008 & 51.2 & 57.7 & 56.7 & 23.6 & 0.01 & 0.006 & 0.442 & 0.361 \\
\hline
\end{tabular}




\section{DISCUSSION}

In the present study we proved that BAN is a disease complex, mainly caused by Fusarium spp., resulting in fruit drop. BAN is the most recently described walnut disease associated with fruit drop in intensive commercial walnut orchards. The most common, and previously reported causes of walnut fruit drop are bacterial blight caused by Xanthomonas arboricola pv. juglandis, codling moth (Cydia pomonella), and pistillate flower abscission (PFA), a fruit drop occurring in the early season due to an excess of pollen on stigmas (González et al. 2008). Bacterial blight is controlled by chemical treatments, while codling moth by integrated codling moth management through mating disruption, whereas PFA cannot be controlled with currently available techniques. Results
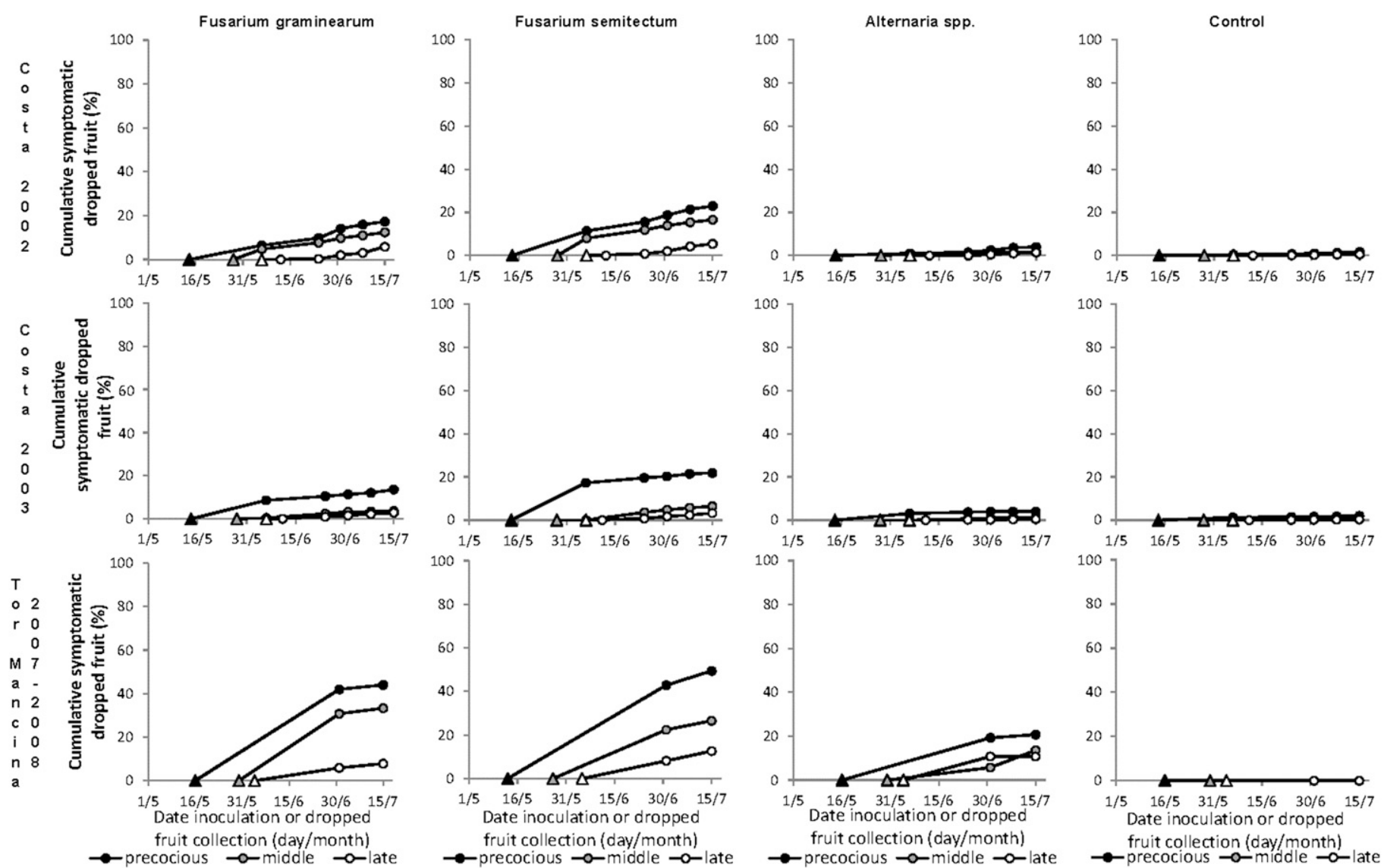

Fig. 4. Cumulative symptomatic fruit drop assessed in artificial inoculation trials performed in two sites (Costa and Tor Mancina), during four years (2002 and 2003 in Costa; 2007 and 2008 in Tor Mancina) and on three inoculation dates (date/month). Triangles show inoculation date, while circles show the cumulative percentages of dropped fruit calculated on the basis of the total number of flowers/fruit present. Values calculated for 15 July correspond to those reported in Table 3.

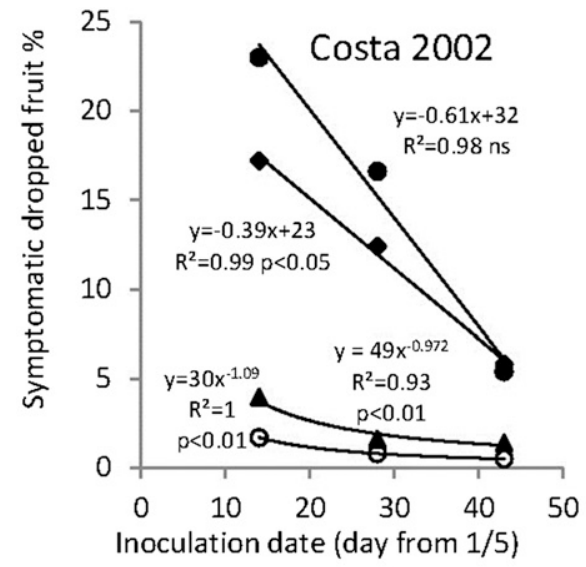

$\bullet$ Fus. gram.

Alt. bulk

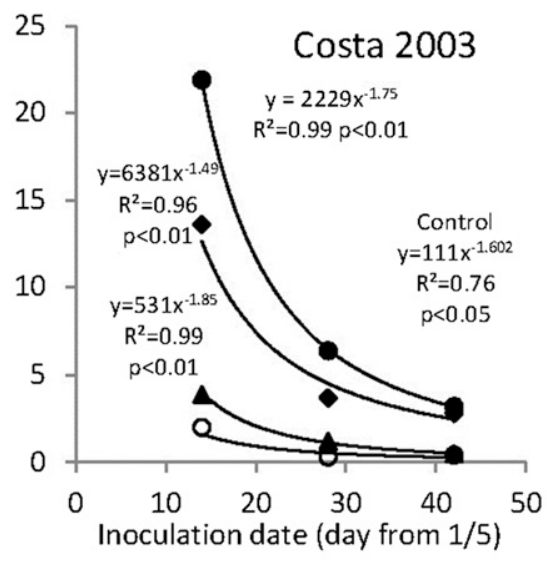

- Fus. gram. $\Delta$ Alt. bulk
- Fus. sem. o Control

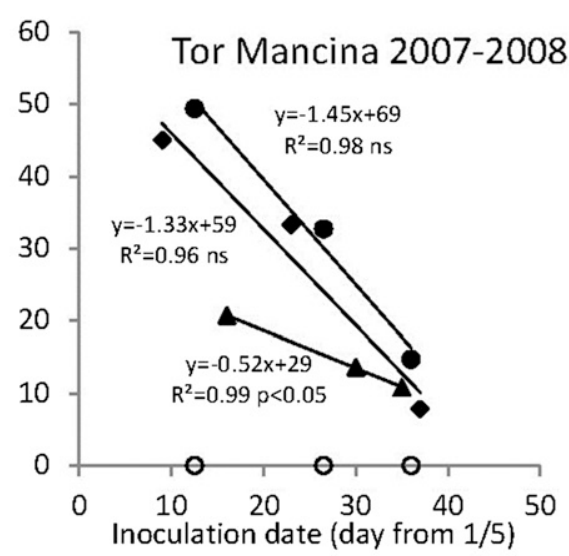

$\bullet$ Fus. gram.

Fus. sem.

$\Delta$ Alt. bulk

o Control

Fig. 5. Total symptomatic fruit drop assessed in artificial inoculation experiments performed in two sites (Costa and Tor Mancina), during 4 years (2002 and 2003 in Costa; 2007 and 2008 in Tor Mancina) after three inoculation dates. Fungal inocula are: Fusarium graminearum (Fus. gram.), Fusarium semitectum (Fus. sem.), and Alternaria spp. (Alt. bulk), respectively. 
obtained in previous studies (Belisario et al. 1999, 2002) allowed to set up a chemical control strategy by which the growers initiated to manage this disease. In this study we considered weather factors and other environmental variables, and phenological stages to identify BAN-conducive factors with the aim also to improve control strategies.

We obtained a clear indication that the common period of multiplication and dispersal of the fungi responsible for BAN (Belisario et al. 2002) coincides to a large extent with the period of maximum susceptibility of walnut to this disease (mid-May to first half of June). This result is extremely important to the timing of chemical treatments against BAN, since no active ingredient with curative control is presently available.

Variables included in the regression model based on environmental characteristics associated with BAN, i.e., interaction of AWR $\times$ relative humidity, maximum temperature (MaxT), and interaction of orchard distance to the closest river/canal (DCR) $\times$ vigor, are mostly representative of climatic or microclimatic conditions occurring in an orchard, and in particular they are related to temperature and humidity. Based upon all results obtained in the present work, the factor which displayed highest influence on the occurrence and severity of BAN fruit drop is maximum temperature and only subordinately humidity. Similar positive effects of maximum temperature on the development of Fusarium disease complex have also been observed in experiments on Fusarium head blight of wheat conducted under controlled environmental conditions using different Fusarium species as inoculant namely, F. graminearum (Xu et al. 2007), and F. culmorum and F. graminearum (Brennan et al. 2005; Rossi et al. 2001).

Highest proportion of variability is explained by the interaction AWR $\times$ relative humidity, which is representative of the orchard natural ventilation condition. In each geographical area, the different orientation of tree rows is due to type of rural land arrangement. From 24 May to 21 June, the main wind direction (but also the second most frequent direction) forms an angle that is significantly lower in the Treviso-Venice area than in Rovigo area (Fig. 7). This condition induces higher ventilation of the orchards located in Treviso-Venice area. The importance of the variable AWR is highlighted also in the literature where it is reported that

TABLE 4. Percentage (\%) of positive recovery either from symptomatic dropped fruit or from randomly sampled fruit collected from inoculated walnut trees

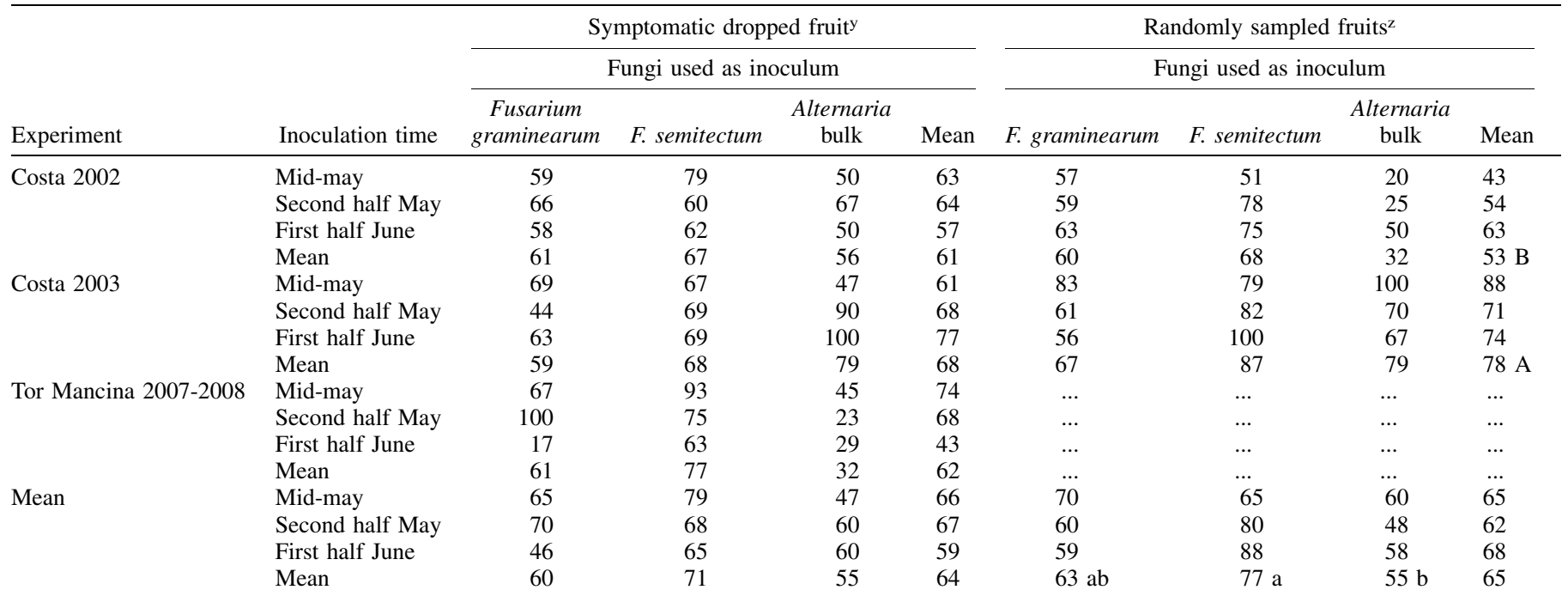

${ }^{y}$ Averaged data on five collection times for Costa and two collection times for Tor Mancina. In symptomatic dropped fruit, nonparametric analysis of variance showed no significant effect for any experimental factor and interaction.

z Averaged data on four collection times. In randomly sampled fruits, main effects of factors. Experiment and inoculated fungus were significant at $P=0.026$ and 0.017 , respectively; and interaction effects were not significant. Values within the same column followed by the same uppercase letter and values within the same row followed by the same lowercase letter are not significantly different $(P=0.05)$.
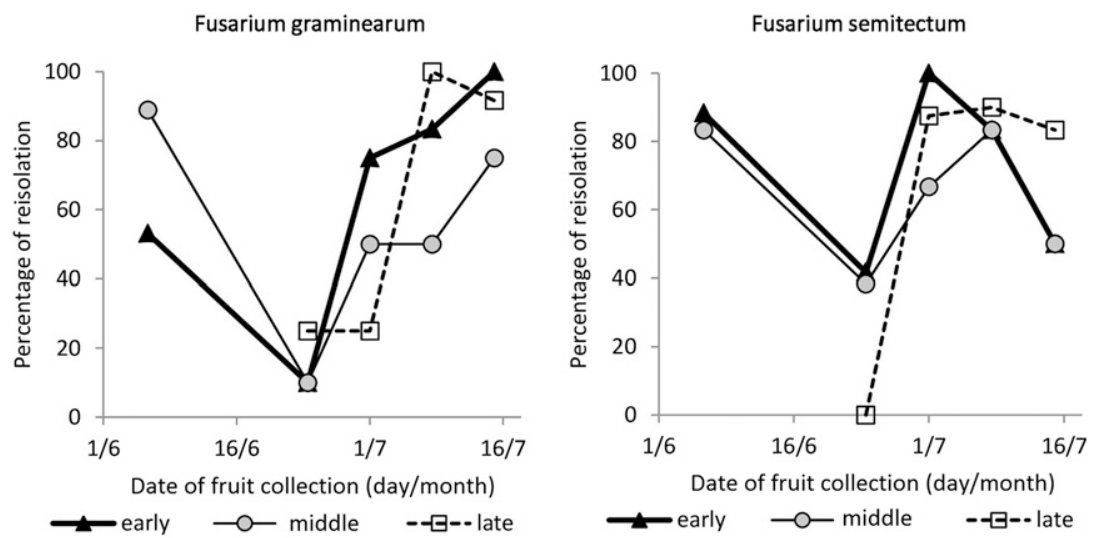

Fig. 6. Trend of positive recovery of Fusarium spp. from symptomatic dropped fruit collected in artificial inoculation experiments performed in Costa during 2 years (2002 and 2003) and at three inoculation dates. Each line is the average trend of the 2 years (2002 and 2003). The mean of the four or five data of each line corresponds to the mean of the two (2002 and 2003) values of the corresponding inoculum/inoculation time reported in Table 4. Data of Alternaria bulk are not graphically represented as they did not show any clear trend. 
during the day, temperature and humidity, particularly within canopy, are higher inside the orchard than outside where there are no trees (Juhász et al. 2011; Oke 1987). The more tree rows are orthogonal to wind direction, the more this effect is evident (Tökei and Dunkel 2003). These observations, associated with the positive correlation between MaxT and fruit drop, suggest that AWR indirectly influences BAN incidence through increasing temperature and humidity inside the orchard in comparison with outside the orchard where there are no trees. The effect of the interaction $\mathrm{AWR} \times$ relative humidity supports the conclusion that the effect of lacking ventilation conducive to BAN is reinforced when relative humidity is high. This may explain higher BAN fruit drop in Rovigo area, where AWR was significantly higher compared with that of orchards located in the Treviso-Venice area.

In the regression analysis, the interaction of the distance to the closest river/canal (DCR) and Vigor significantly affected fruit drop, further suggesting the importance of humidity in fruit drop development, which is higher in areas closer to water surfaces. Similarly, humidity effects have also been observed in experiments performed on Fusarium head blight by Xu et al. (2007). However, the amount of variability explained by this latter regression term is clearly less than by AWR $\times$ relative humidity and MaxT. In any case, walnut fruit are susceptible to BAN only during a 3- to 4-week span of time of their physiological development. Environmental conditions have to be conducive during this limited period for successful establishment of BAN infection.

Walnut genotype, orchard age, and leaf wetness can also be important factors affecting the occurrence and severity of BAN (Belisario et al. 2002). Leaf wetness was not directly involved in our regression analyses since weather instrument shelters were located outside the orchards. However, as explained above, the significant effect found for AWR and orchard distance to the closest river/canal (DCR) is probably associated with leaf wetness, suggesting its positive influence on BAN occurrence.

The inoculation method we developed in this study allowed the inoculated plants to develop all stages of disease symptoms, whereas it was not possible to observe such development of all disease symptoms in previous works where inoculations were carried out in the greenhouse on flowers and fruit detached from plants which rapidly deteriorated (Belisario et al. 2002). Inoculations with the three fungi all resulted in significant fruit drop compared with noninoculated control plants in all years and locations examined. In noninoculated control trees, symptomatic fruit drop was very low, $0.6 \%$ on average. This is because fruit drop of noninoculated fruit is related not only to weather factors that were not conducive to BAN infection in years 2002 and 2003, but also to the availability of natural inoculum which was very low in Costa because of chemical treatments applied to the remaining part of the farm. In Costa, BAN occurrence in 2003 might have been hampered by drought recorded from late spring through the summer. In Tor Mancina, BAN had never been observed in sampled walnut orchards in the years before experimentation due to the absence of inoculum and/or conducive environmental factors.

Reisolations from inoculated symptomatic fruit resulted in $60 \%$ recovery of the fungi, even though percentages varied during the assessment period with the lowest values corresponding to the second half of June (Fig. 6). The low recovery, which was common to both Fusarium and Alternaria, might be due to the fruit developmental stage at which phenolic content in the fruit is high (Solar et al. 2006). In fact, the phenolic content (in particular juglone concentration) is particularly high in walnut fruit around the second half of June, before husk lignification, and phenols are known to have inhibitory or lethal effects against fungi and bacteria (Solar et al. 2006).

F. semitectum was observed to cause browning and shriveling when flowers and young fruit were inoculated and the damage was higher in early inoculation than late inoculations. This characteristic makes damage caused by $F$. semitectum underestimated since dropped necrotic flowers/fruit were not considered as symptomatic when visually examined more than 3 days after dropping, being instead included in the necrotic/mummified fruit category. Though the higher fruit drop caused by F. semitectum was not significantly different from that caused by $F$. graminearum, the former pathogen can be considered more damaging for its capacity to induce a vast type of lesions. This fact can partially explain the greater damage from natural infection observed in Rovigo area, where the presence of this fungus was higher than that in Treviso-Venice area (Belisario et al. 2002).

Results of this study contribute to a better understanding of the etiology and epidemiology of BAN. The findings on the extended phenological time span of walnut flowers/fruit susceptibility to $\mathrm{BAN}$ and the environmental factors conducive to BAN development can be useful in adjusting cultural practices and timing of chemical treatments to control BAN. Farmers will need to consider management strategies aimed at protecting flowers/fruit from infection for several weeks rather than several days around flowering in case of occurrence of conducive environmental factors.

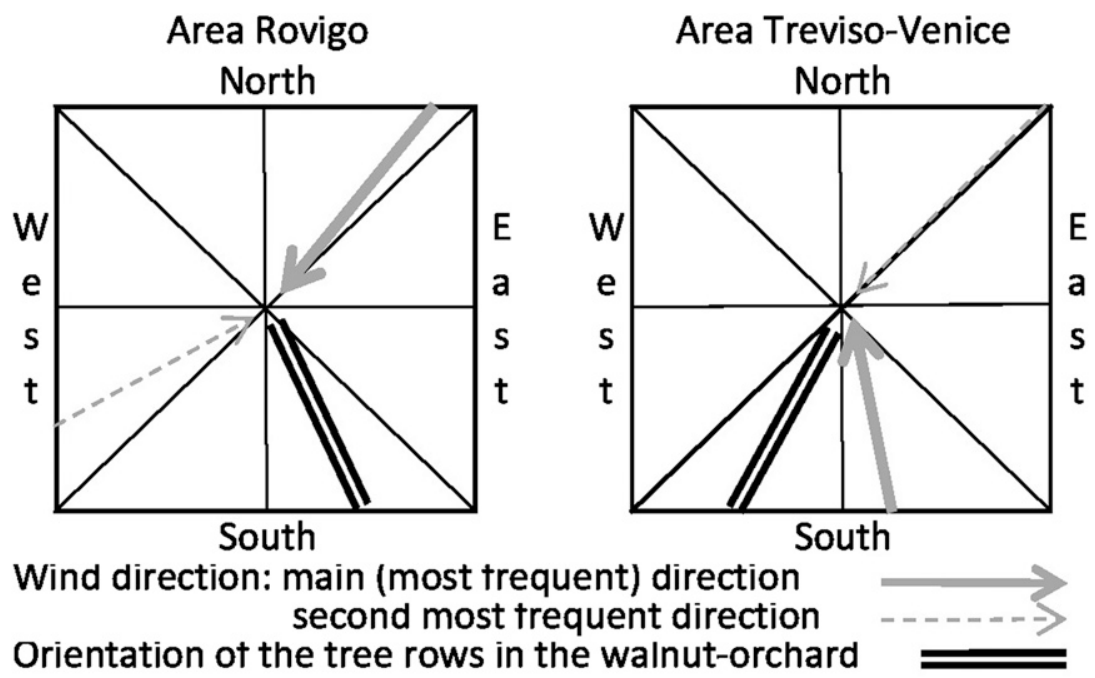

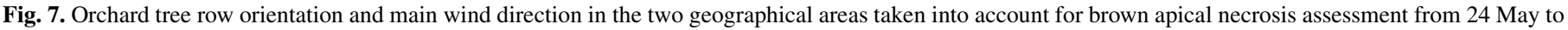
21 June 2002 to 2003. 


\section{ACKNOWLEDGMENTS}

We thank M. Maccaroni and A. Santori for the precious scientific support in the delicate phase of in planta artificial inoculation experiments carried out in Costa and Tor Mancina; S. Sibbet for his thorough revision of the manuscript and for his precious suggestions; and the walnut growers who financially supported the research and collaborated in the organization and execution of experimental trials and fruit drop data recording. We also thank the Regional Agency for Environmental Prevention and Protection of Veneto (ARPAV), the Regional Agency for the Development and Innovation in Agriculture of Lazio (ARSIAL), and the Research Centre for the study of the Plant-Soil Relations (CRA-RPS) for providing the meteorological data.

\section{LITERATURE CITED}

Anderson, M. J., and Ter Braak, C. 2003. Permutation tests for multi-factorial analysis of variance. J. Stat. Comput. Simul. 73:85-113.

Belisario, A., Balmas, V., Santori, A., Valier, A., and Corazza, L. 2001. Fusarium necrosis on Persian (English) walnut fruit. Acta Hortic. 544: 389-393.

Belisario, A., Forti, E., Balmas, V., Valier, A., and Corazza, L. 1999. La fusariosi del frutto di noce comune. Inf. Agrario 21:51-52.

Belisario, A., Maccaroni, M., Coramusi, A., Corazza, L., Figuli, P., and Pryor, B. M. 2004. First report of Alternaria species groups involved in disease complexes of hazelnut and walnut fruit. Plant Dis. 88:404.

Belisario, A., Maccaroni, M., Corazza, L., Balmas, V., and Valier, A. 2002. Occurrence and etiology of brown apical necrosis on Persian (English) walnut fruit. Plant Des. 86:599-602.

Belisario, A., Santori, A., Potente, G., Fiorin, A., Saphy, B., Reigne, J. L., Pezzini, C., Bortolin, E., and Valier, A. 2010. Brown apical necrosis (BAN): A fungal disease causing fruit drop of English walnut. VI International Walnut Symposium. ISHS Acta Hort. 861:449-452.

Belsley, D. A., Kuh, E., and Welsch, R. E. 1980. Regression Diagnostics. John Wiley \& Sons, New York.

Borrego-Benjumea, A., Basallote-Ureba, M. J., Melero-Vara, J. M., and Abbasi, P. A. 2014. Characterization of Fusarium isolates from asparagus fields in Southwestern Ontario and influence of soil organic amendments on Fusarium crown and root rot. Phytopathology 104:403-415.

Brennan, J. M., Egan, D., Cooke, B. M., and Doohan, F. M. 2005. Effect of temperature on head blight of wheat caused by Fusarium culmorum and F. graminearum. Plant Pathol. 54:156-160.
Corpas-Hervias, C., Melero-Vara, J. M., Molinero-Ruiz, M. L., Zurera-Munoz, C., and Basallote-Ureba, M. J. 2006. Characterization of isolates of Fusarium spp. obtained from asparagus in Spain. Plant Dis. 90:1441-1451.

Durbin, J., and Watson, G. S. 1951. Testing for serial correlation in least squares regression, II. Biometrika 38:159-179.

Edgington, E. S., and Onghena, P. 2007. Randomization Tests, 4th ed. Chapman and Hall/CRC Press, Boca Raton, FL.

Elmer, W. H. 1996. Fusarium fruit rot of pumpkin in Connecticut. Plant Dis. $80: 131-135$.

González, C. R., Lemus, G. S., and Reginato, G. 2008. Pistillate flower abscission symptoms of 'Sere' walnut (Juglans regia L.). Chil. J. Agric. Res. 68:183-191.

Hong, S. G., Maccaroni, M., Figuli, P. J., Pryor, B. M., and Belisario, A. 2006. Polyphasic classification of Alternaria spp. isolated from hazelnut and walnut in Europe. Mycol. Res. 110:1290-1300.

Juhász, Á., Sepsi, P., and Tókei, L. O. 2011. Micrometeorological measurements in orchard and above bare soil. Acta Universitatis Sapientiae. Agric. Environ. 3:93-101.

Klix, B. M., Beyer, M., and Verreet, J. A. 2008. Effects of cultivar, agronomic practices geographic location, and meteorological conditions on the composition of selected Fusarium species on wheat heads. Can. J. Plant Pathol. 30:46-57.

Oke, T. R. 1987. Boundary Layer Climates. Routledge, London, New York.

Osborne, J. W. 2010. Improving your data transformations: Applying the BoxCox transformation. Pract. Assess. Res. Eval. 15:1-9.

Parry, D. W., Jenkinson, P., and McLeod, L. 1995. Fusarium ear blight (scab) in small grain cereals-A review. Plant Pathol. 44:207-238.

Pillar De Patta, V. 2006. MULTIV. Multivariate Exploratory Analysis, Randomization Testing and Bootstrap Resampling. User's Guide v. 2.4.Universidade Federal do Rio Grande do Sul, Porto Alegre, Brazil.

Rossi, V., Ravanetti, A., Pattori, E., and Giosuè, S. 2001. Influence of temperature and humidity on the infection of wheat spikes by some fungi causing Fusarium head blight. J. Plant Pathol. 83:189-198.

Solar, A., Colaric, M., Hudina, M., and Stampar, F. 2006. Phenolic content of walnut fruit as affected by cultivar and developmental stage. Acta Hortic. 705:231-240.

Tökei, L., and Dunkel, Z. 2003. Investigation of crop canopy temperature in apple study orchard. Abstracts from the meeting. EGS-AGU-EUG Joint Assembly, Nice, France.

White, H. 1980. A heteroskedasticity-consistent covariance matrix estimator and a direct test for heteroskedasticity. Econometrics 48:817-838.

Xu, X.-M., Monger, W., Ritieni, A., and Nicholson, P. 2007. Effect of temperature and duration of wetness during initial infection periods on disease development, fungal biomass and mycotoxin concentrations on wheat inoculated with single, or combinations of, Fusarium species. Plant Pathol. 56:943-956. 